\title{
RESULTS OF THE RECONSTRUCTED MEDEBIS
}

\author{
R. Becker, $\underline{\text { H. Hoeltermann, M. Kleinod }}$ \\ Institut fuer Angewandte Physik, \\ J.W.Goethe-Universitaet, D-60054 Frankfurt/Main
}

\begin{abstract}
The electron beam ion source (EBIS) can deliver sufficiently short and intense pulses of fully stripped light ions for single turn injection into a dedicated synchrotron for hadron therapy. The technology of such a MEDEBIS resembles travelling wave tubes used in satellites and can also be designed for lifetimes up to ten years. In a first setup using a normal conducting solenoid of $0.75 \mathrm{~T}$ and a trap length of $0.25 \mathrm{~m}$ the source delivered high charge states of light ions like $\mathrm{O}^{8+}, \mathrm{O}^{7+}, \mathrm{O}^{6+}$ and $\mathrm{C}^{6+}, \mathrm{C}^{5+}[1]$. The fast ion extraction in less than $2 \mu$ s is realised trough a special electrostatic structure providing a pulsed extraction potential gradient along the axis of the whole ion trap. The first results were very promising, but the residual gas pressure in the ionisation region was too high to make use of a sufficiently long confinement time in order to reach full abundance of bare nuclei. For the reconstructed MEDEBIS the inner two windings of the solenoid were drilled out to allow for a vacuum tube of about twice the diameter with better conductance. Together with the implementation of NEG getter material the residual gas pressure should be lowered sufficiently to reach the required yield of the fully stripped ions. Iron disks of high permeability have been added at both ends of the solenoid to increase the homogeneity of the magnetic field as well as to reduce the field in the gun and collector regions for a higher focussed current density in the trap region. The new MEDEBIS is under test and new experimental results will be presented.
\end{abstract}

\section{INTRODUCTION}

Treating cancers with heavy ions is a very promising kind of therapy for cases in which local control of tumors with conventional therapy fails. Conventional radiation such as bremsstrahlung, electrons or $\gamma$-rays show an exponential decrease of deposited energy while penetrating deeper into tissue. Ions in contrary deposit most of their energy in a narrow region, the bragg peak, at the end of their track, so healthy tissue in the tumor's vicinity will not being radiated. The first approaches to this kind of therapy have taken place since 1994 using large research accelerator centers i.e. Berkeley, United States, Chiba, Japan or GSI, Germany. Their aim is to give proof-of-principle while the realisation of hospital based therapy will need smaller machines dedicated only to clinical purposes. This is the point where an electron ion beam source (EBIS) comes into consideration. An EBIS yields a short, but very intense ion-pulse of fully stripped ions. Using an EBIS gives sufficiently current of bare nuclei so the ion source alone combined with a low duty cycle RFQ is all what is needed as an injection line for a synchrotron[2]. Even the time structure of the ion pulse is well suited for single turn injection, leading to a less complex synchrotron. Advantageously, the lifetime of an EBIS, resembling travelling wave tubes in satellites, can easily reach ten years since it has no consumable parts. A setup like this wouldn't need a linac besides a RFQ and a stripper[3].

\section{THE MEDEBIS PRINCIPLE}

At the Institut fuer Angewandte Physik, GoetheUniversitaet, Frankfurt/Germany we assembled last year a reconstructed prototype of such an medically dedicated EBIS, named MEDEBIS. To reduce complexity and costs we use a normal conducting solenoid of $0.8 \mathrm{~T}$ for magnetical focussing of the electron beam. The electron gun and collector are magnetically shielded by iron cylinders of high permeability. Within the electron beam gas atoms of the feed gas like oxygen or carbon (methane) are getting ionised due to electron impact. Ions will be trapped radially in the space charge of the electron beam while they are confined on a length of $0.25 \mathrm{~m}$ due to the positive potential of barrier electrodes around the axis. For the extraction a potential gradient will be applied to the ion trap, provided by a combination of two reverse slitted electrostatic structures on different radius. The slits have a parabolic shape resulting in an adjustable linear gradient while having different potentials on each electrode. The rise of the gradient allows a pulse compression down to $2 \mu$ s, what has already been proofed[4]. The confinement time of the ions is dependent on the charge state and number of bare nuclei, so ion species and current can be adjusted by the frequency of pulsing. A very important fact for the EBIS principle is the vaccum condition inside of the ion trap. As most of the currently used EBIS devices base on a cryogenic environment we had to deal with the higher desorption rates of a warm solenoid. So far we installed a larger vaccum tube in which NEG getter material has been introduced to provide an active pumping surface. 
The expected ion current being trapped in the electron beam is limited by the space charge density $\rho$ of the beam. Experience so far has shown that the number of extracted ion charges cannot exceed the number of electrons in the trap region. With $\mathrm{N}^{-}$the number of electrons in a cylindrical electron beam of the length 1 , perveance $\mathrm{P}$ and the energy e $\mathrm{U}_{0}$, the number of storeable ions $\mathrm{N}^{+}$is equal or less than the number of electrons:

$$
\mathrm{N}^{+} \leq \frac{\mathrm{N}^{-}}{\mathrm{q}}=\sqrt{\frac{\mathrm{m}_{\mathrm{e}}}{2 \mathrm{e}^{3}}} \cdot \frac{\mathrm{P} \cdot 1 \cdot \mathrm{U}_{0}}{\mathrm{q}} \cong \frac{10^{13}}{\mathrm{q}} \cdot \mathrm{P} \cdot 1 \cdot \mathrm{U}_{0}
$$

Tab. 1: Actual MEDEBIS parameters:

\begin{tabular}{|l|l|}
\hline Geometry & \\
\hline Length of ion trap & $0.25 \mathrm{~m}$ \\
\hline Magnetic field & $0.8 \mathrm{~T}$ \\
\hline Electron beam & \\
\hline Energy & $3 \mathrm{keV}$ \\
\hline Electron current & $300 \mathrm{~mA}$ \\
\hline Current density & $\sim 250 \mathrm{~A} / \mathrm{cm}^{2}$ \\
\hline Extraction & \\
\hline $\mathrm{C}^{6+}$ particles per spill* & $2.2^{*} 10^{10}$ \\
\hline Shortest extraction time & $2 \mu \mathrm{s}$ \\
\hline Ion current $\mathrm{C}^{6+}$ & $1.5 \mathrm{~mA}$ \\
\hline Extraction gradient & $0-3 \mathrm{kV} / \mathrm{m}$ \\
\hline
\end{tabular}

Calculated by (1) for maximum of storeable charges at $2 \mu$ s extraction time at present working parameters. Not yet being observed!

\section{MEASUREMENTS AND RESULTS}

Due to the extensive use of passive magnetic elements such as iron discs enclosing the electron gun and the main solenoid two important improvements have been done. First of all the magnetic field along the ion trap shows a more constant behaviour over a broader length than before, which is equal to a constant high compression in this area.

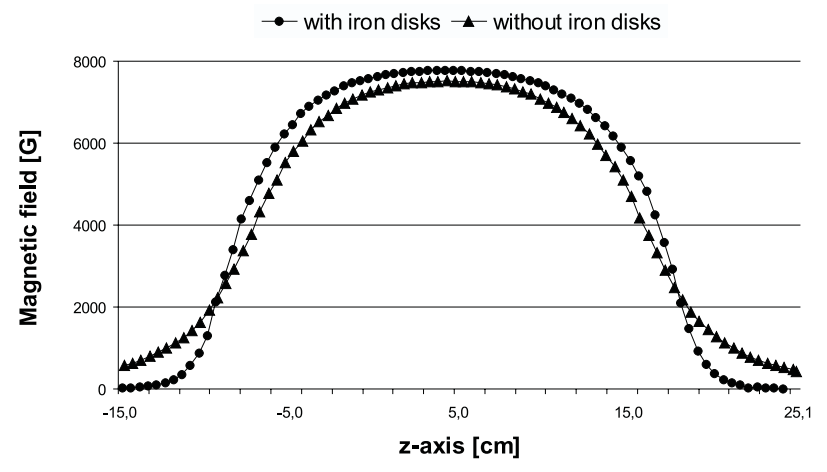

Fig 1.: MEDEBIS magnetic field along the ion trap
Secondly a higher beam compression right after the cathode leads to an more intensive electron beam. The electron current which could be obtained up to now is $300 \mathrm{~mA}, 80 \%$ more than in the first setup. Ionisation measurements at $65 \mathrm{~mA}$ allow to deduce a current density of $50 \mathrm{~A} / \mathrm{cm}^{2}$, what leads to the conclusion that with the unchanged parameters of the main solenoid and given apperture of the barrier electrodes we have now current densities around $250 \mathrm{~A} / \mathrm{cm}^{2}$. Measurement of the actual current density through charge state breeding is already in process. The whole setup has been constructed for currents up to $800 \mathrm{~mA}$ and current densities in the region of $600 \mathrm{~A} / \mathrm{cm}^{2}$, leading to particle spills of $3^{*} 10^{10} \mathrm{C}^{6+}$ or an ion current equivalent to $\sim 2 \mathrm{~mA}$. During the ramp up of the electron beam it has been observed, that a dynamic reconfiguring of the magnetic fields especially at the end of the ion trap is necessary, while increasing the beam current. This can be interpreted as a change of the beam spreading due to its increasing space charge at constant magnetic focussing which needs a different magnetic field to be counteracted.

Loss of electron current on some of the electrodes could be minimized through the steerable iron shielding discs as well as through a reconfigured magnetic field. The new developed collector designed for surpressing of $\mathrm{x}$-rays leading to desorption as well as to cope with a $4 \mathrm{~kW}$ electron beam has already collected $96 \%$ of the $1 \mathrm{~kW}$ electron beam, which is currently under test. During all experiments a pressure of $10^{-9}$ mbar in the electron gun and collector recipients is maintained.

\section{BEAM PURITY}

The medical application of the ion beam makes beam impurity an important issue. Different ion species will cause unwanted shaping of spread out bragg peaks resulting in different biological effects. All ion sources usually deliver an ion spectrum with a mixture of charge states and species. This is especially a problem using ions of the same charge to mass ratio. At the GSI proposed facility[5] magnetically selected $\mathrm{C}^{4+}$ ions will be accelerated up to the injection energy of the synchrotron where they will pass a stripper to remove the final two electrons. By this procedure bare nuclei of nitrogen or oxygen are not accepted simultaneously by the synchrotron, resulting in a very low impurity admixture. Of course an EBIS could also be operated at lower confinement time or current density delivering now a spectrum of ions peaking at $\mathrm{C}^{4+}$. The lower relative abundance necessitates a higher loading of the source capacity which could be counteracted by a higher electron current, which is easy at lower current density. Another possibility would be the use of isotopes like $\mathrm{C}^{13+}$, neglecting the use of PET for in-situ monitoring, or the implementation of ion cyclotron resonance[6] for ion separation during breeding of higher charge states. 


\section{REFERENCES}

[1] O.Kester, R. Becker and M. Kleinod, Rev. Sci. Instrum. 67, 1165 (1996)

[2] R. Becker, M. Kleinod, A. Schempp, E.D. Donets, and A.I. Pikin, Rev. Sci. Instrum. 63 (1992) 2812

[3] R. Becker, H. Hoeltermann, M. Kleinod, A. Schempp, B. Zipfel, Proc. EPAC 98, Stockholm 22-26 Juni 98, to be publ.

[4] M. Kleinod, R. Becker, H. Hoeltermann, M. Muecke, R. Rao, M. Weidenmueller, and B. Zipfel Rev. Sci. Instrum. 69 (1998) 718

[5] Proposal for a dedicated ion beam facility for cancer therapy, GSI Darmstadt/Germany, September 1998

[6] B. Zipfel, R. Becker, M. Kleinod, and R. Rao Proc. 3 rd Euroconference on Atomic Physics with Stored Highly Charged Ions, Ferrara, Italy, 22-26 Sept. 1997, Hyperfine Interactions 115 (1998) p. $193-200$ 